\title{
Erratum a: Terapia sostitutiva combinata con L-T4 e L-T3 nell'ipotiroidismo
}

\author{
Bernadette Biondi $^{1}$ - Francesco S. Celi ${ }^{2}$ Leonidas Duntas ${ }^{3}$. Andrea M. Isidori ${ }^{4}$. \\ Alfredo Pontecorvi ${ }^{5}$ - Domenico Salvatore ${ }^{6}$. Paolo Vitti ${ }^{7} \cdot$ Francesco Trimarchi $^{8}$
}

Pubblicato online: 1 settembre 2016

(C) Springer International Publishing AG 2016

Erratum a: L'Endocrinologo (2015) 16(6): 264-268

DOI 10.1007/s40619-015-0158-6

La presente Tabella sostituisce quella già pubblicata, essendo intervenute, nelle more della pubblicazione, variazioni relative alla disponibilità commerciale di alcune preparazioni farmaceutiche.

La versione online dell' articolo originale può essere trovata al doi:10.1007/s40619-015-0158-6.

Materiale elettronico supplementare La versione elettronica di questo articolo (DOI:10.1007/s40619-016-0207-9) contiene materiale supplementare, disponibile per gli utenti autorizzati.

$\triangle$ B. Biondi

bebiondi@unina.it; bebiondi@libero.it

1 Dipartimento di Endocrinologia Clinica e Chirurgia, Università Federico II, Napoli, Italia

2 Division of Endocrinology and Metabolism, Department of Internal Medicine, Virginia Commonwealth University School of Medicine, Richmond, VA, USA

3 Evgenidion Hospital, Thyroid Unit, University of Athens, Athens, Greece

4 Dipartimento di Medicina Sperimentale, Sapienza Università di Roma, Roma, Italia

5 Unità di Endocrinologia, Università Cattolica del Sacro Cuore, Roma, Italia

6 Dipartimento di Medicina Clinica e Chirurgia, Università Federico II, Napoli, Italia

7 Dipartimento di Medicina Clinica e Sperimentale, Unità 1 di Endocrinologia, Università di Pisa, Pisa, Italia

8 Accademia Peloritana dei Pericolanti, Università di Messina, Messina, Italia 